\title{
Effects of vitamin A deficiency on mitochondrial function in rat liver and heart
}

\author{
Ernesto Estornell ${ }^{1}$, José R. Tormo ${ }^{1}$, Pilar Marín ${ }^{1}$, Jaime Renau-Piqueras ${ }^{2}$, Joaquín Timoneda ${ }^{1}$ and \\ Teresa Barber ${ }^{1 *}$ \\ ${ }^{1}$ Department de Bioquímica Biologia Molecular, Facultat de Farmàcia, Universitat de València, Spain \\ ${ }^{2}$ Centro de Investigación, Hospital La Fé, València, Spain
}

(Received 2 February 2000 - Revised 11 July 2000 - Accepted 19 July 2000)

\begin{abstract}
The aim of this study was to investigate comparative effects of vitamin A deficiency on respiratory activity and structural integrity in liver and heart mitochondria. Male rats were fed a liquid control diet (control rats) or a liquid vitamin A-deficient diet (vitamin A-deficient rats) for 50 days. One group of vitamin-A deficient rats was refed a control diet for 15 days (vitamin Arecovered rats). To assess the respiratory function of mitochondria the contents of coenzyme $\mathrm{Q}$ (ubiquinone, CoQ), cytochrome $c$ and the activities of the whole electron transport chain and of each of its respiratory complexes were evaluated. Chronic vitamin A deficiency promoted a significant increase in the endogenous coenzyme Q content in liver and heart mitochondria when compared with control values. Vitamin A deficiency induced a decrease in the activity of complex I (NADH-CoQ reductase) and complex II (succinate-CoQ reductase) and in the levels of complex I and cytochrome $c$ in heart mitochondria. However, NADH and succinate oxidation rates were maintained at the control levels due to an increase in the CoQ content in accordance with the kinetic behaviour of CoQ as an homogeneous pool. On the contrary, the high CoQ content did not affect the electron-transfer rate in liver mitochondria, whose integrity was preserved from the deleterious effects of the vitamin A deficiency. Ultrastuctural assessment of liver and heart showed that vitamin A deficiency did not induce appreciable alterations in the morphology of their mitochondria. After refeeding the control diet, serum retinol, liver and heart CoQ content and the activity of complex I and complex II in heart mitochondria returned to normality. However, the activities of both whole electron transfer chain and complex I in liver were increased over the control values. The interrelationships between physiological antioxidants in biological membranes and the beneficial effects of their administration in mitochondrial diseases are discussed.
\end{abstract}

Vitamin A deficiency: Coenzyme Q (ubiquinone): Mitochondrial respiratory chain

Vitamin $\mathrm{A}$ is a generic term which describes a number of molecules exhibiting the biological activity of retinol, the precursor of naturally-occurring retinoids. Although vitamin A was one of the first vitamins to be discovered, the full range of its activities remains to be defined. Vitamin A (all-trans-retinol) and its natural cis and trans derivatives are involved in important physiological functions: for example, vision and growth, immunity, embryological development and cell differentiation; and also as physiological antioxidants with a great potential role in the prevention of several diseases related with oxidative stress that are in continuous research (Ross, 1992; Livrea \&
Tesoriere, 1998; Zile, 1998; Palace et al. 1999; Semba, 1999).

The antioxidant properties of some retinoids, known for decades, have been reinvestigated in recent years in chemical as well as in biological systems. Its lipid nature and localization within the lipophilic compartment of membranes and lipoproteins make vitamin A effective in reducing lipid peroxidation by acting as a chain-breaking antioxidant. The extremely low concentration of oxidised products of vitamin A, as retinoic acid, and its comparative low antioxidant activity, make it an unlikely factor in modulating cellular oxidative stress, although it is now

\footnotetext{
Abbreviation: CoQ, coenzyme Q

* Corresponding author: Teresa Barber, fax +34 6 3864917, email Barber@uv.es
} 
known that many of the vitamin A effects are mediated, via retinoic acid isomers, on gene transcription. In fact, antioxidant activities of retinoids have been ranked as retinol $\geq$ retinal $\gg$ retinyl palmitate $>$ retinoic acid (Livrea \& Tesoriere, 1998; Palace et al. 1999).

In a previous work we reported that heart mitochondria from vitamin A-deficient rats showed a defect in the respiratory complex I (NADH-coenzyme Q (CoQ) reductase activity; Estornell et al. 1997), which is the most complex among the proton translocating enzymes of mitochondria and contains the highest number of mitochondrial-encoded subunits. Complex I functional defects are implicated in a number of congenital and acquired pathologies, even the normal ageing process could be associated to them (Schapira, 1998; Wallace, 1999). Mitochondria have been recognised as a major physiological source of reactive oxygen species, which arise as a consequence of oxygen reduction. The presence of several electron carriers and polyunsaturated fatty acid rich membranes makes this organelle highly susceptible to free-radical attack leading to alterations of its structural integrity and functions. In this sense, free-radical mechanisms have also been implicated in the liver mtDNA damage produced in vitamin A-deficient rats (T Barber et al., Free Radical Biology and Medicine, in press).

The aim of this study was to compare the effects of vitamin A deficiency and their reversibility on mitochondrial respiratory activity and mitochondrial structural integrity in two organs with different rates of oxygen consumption, liver and heart.

\section{Materials and methods}

\section{Chemicals}

Decylubiquinone, rotenone, antimycin A, cytochrome $c$ (from horse heart, type III) and other biochemical reagents were purchased from Sigma Chemical Co. (St. Louis, MO, USA). Salts and solvents were from Merck (Darmstadt, Germany). Diet components were from ICN Biomedicals (Cleveland, OH, USA) and Sigma Chemical Co. Decylubiquinol was formed by reducing decylubiquinone. CoQ analogues and inhibitors were kept as ethanol solutions in the dark, and their titres were determined spectrophotometrically (Lenaz et al. 1995b; Estornell et al. 1993). Ferrous cytochrome $c$ was obtained as described previously (Degli Esposti \& Lenaz, 1982).

\section{Animals and diets}

Male rats were made deficient in vitamin A by feeding a liquid vitamin A-free diet based on the formulations given previously (Estornell et al. 1994, 1997; Barber et al. 1999) with the recommendations of the American Institute of Nutrition (Reeves et al. 1993), as follows. Five pregnant rats (Charles River, Barcelona, Spain) were housed in individual cages in a room maintained at $22^{\circ} \mathrm{C}$ with a $12-\mathrm{h}$ light-dark cycle. After pup birth, the dams with their litter were randomly divided into two groups. The first group (two dams with their pups) was fed on a complete semisynthetic liquid diet (Barber et al. 1999) but sunflower oil, which contains less vitamin traces than others, was used as the unique lipid source in order to better control the vitamin A content of the diet (C. Ross, personal communication). The composition of this diet ( $\mathrm{g} / \mathrm{l})$ was, in distilled deionised water: casein (vitamin-free) 54.5, DL-methionine $0 \cdot 8$, dextrin $162 \cdot 7$, sunflower oil $13 \cdot 0$, AIN-93 vitamin mix 2.6, AIN-93G mineral mix 9.2, choline chloride $0 \cdot 4$, cellulose powder $10 \cdot 0$, xantham gum $2 \cdot 0$. The composition of AIN-93 vitamin mix ( $\mathrm{g} \%$ ) was: niacin, 0-300; D-calcium pantothenate, $0 \cdot 160$; pyridoxine $\mathrm{HCl}, 0 \cdot 070$; thiamine $\mathrm{HCl}$, 0.060; riboflavin, 0.060; folic acid, 0.020; biotin, 0.002; vitamin $\mathrm{B}_{12}, 0.250$; all-trans-retinyl palmitate $(250000 \mathrm{U} /$ g), 0.160; $\alpha$-tocopherol powder $(250 \mathrm{U} / \mathrm{g}), 3 \cdot 000$; vitamin $\mathrm{D}_{3} \quad(400000 \mathrm{U} / \mathrm{g}), 0.025$; menadione, 0.008; powder sucrose, $95 \cdot 885$. The energy content of this diet was 4.18 MJ/l. The second group (three dams with their pups) was fed on the same diet but devoid of vitamin A. Feeding dams on the vitamin A-free diet during the $21 \mathrm{~d}$ of lactation shortens the interval of vitamin A depletion in the pups by reducing its transfer during the suckling period (Pasatiempo et al. 1991). At $21 \mathrm{~d}$ eight male pups from mothers fed the complete diet (control group) and thirteen male pups from mothers fed the vitamin A-free diet (deficient group) were weaned into their corresponding mother diet until they were $50 \mathrm{~d}$ old. This period was appropriate to induce a severe deficiency in vitamin A without significantly altering the growth of the animals. At the end of the deficiency treatment five male vitamin A-deficient rats were subsequently fed on the complete diet during $15 \mathrm{~d}$ to induce a repletion of vitamin A (recovered group). This group was used to study the reversibility of the possible changes caused by the vitamin deficiency. The animals not used in this study were returned to the control diet. All diets were made daily. The amount of food intake was not significantly different between groups.

\section{Sampling procedure}

The experiments were performed between 10:00-12:00 a.m. Rats were anaesthetised with pentothal $(50 \mathrm{mg} / \mathrm{kg}$ body weight, intraperitoneally). Blood was collected from the aorta in heparinised syringes and then liver and heart samples were taken and processed immediately.

\section{Retinoid and tocopherol determination}

Serum vitamin A concentration was determined by the spectrofluorimetric method of Selvaraj \& Susheela (1970) using retinol palmitate as standard. The different forms of vitamin A (retinol, retinoic acid and retinyl esters), $\beta$ carotene and tocopherols were measured in serum and tissues following the method described by Barua \& Olson (1998).

\section{Mitochondria isolation}

Liver and heart mitochondria were isolated as described previously (Johnson \& Lardy, 1967; Smith, 1967). 


\section{Coenzyme $Q$ assay}

The CoQ content of homogenates and mitochondrial membranes was measured according to Kröger with minor modifications (Kröger, 1978). Liver and heart homogenates and isolated mitochondria were diluted to $1 \mathrm{ml}$ in sucrose $(250 \mathrm{mM})$, Tris- $\mathrm{HCl}$ buffer $(10 \mathrm{mM}$; $\mathrm{pH}$ $7.4)$, and they were extracted with methanol-light petroleum $(60: 40 \mathrm{v} / \mathrm{v})$. After collection of the upper layers, the residues were re-extracted twice with light petroleum. The combined extracts were evaporated under nitrogen stream and the dry residues were dissolved in ethanol. The CoQ content was determined in the ethanolic solutions by recording the absorbance at $280 \mathrm{~nm}$ minus the absorbance at $289 \mathrm{~nm}$ caused by reduction with K-borohydride and by oxidation in the presence of $\mathrm{KOH}(\varepsilon=8 \cdot 8 / \mathrm{mM}$ per $\mathrm{cm})$.

\section{Respiratory chain enzymatic activity determination}

Individual and integrated enzymatic activities were assayed at $22^{\circ} \mathrm{C}$ in potassium phosphate buffer, $(50 \mathrm{~mm}$; and $\mathrm{pH}$ 7.4), EDTA (1 mM). When appropriate, potassium cyanide $(2 \mathrm{mM})$ was added. Isolated liver and heart mitochondria were diluted to $15-25 \mu \mathrm{g} / \mathrm{ml}$ in the cuvette after freezing and thawing, which was found to completely remove the permeability barrier to NADH without significant damage to the respiratory enzyme activities (Lenaz et al. 1995a; Genova et al. 1995). Quinones and inhibitors were added in ethanolic solution at a maximum of $2 \%$ ethanol in the assay mixture.

Integrated aerobic NADH oxidation and $\mathrm{NADH}-\mathrm{CoQ}$ reductase activity (complex I) were assayed as previously described (Estornell et al. 1993). The soluble saturatedstraight-chain decylubiquinone was used as ubiquinone analogue. Succinate-CoQ reductase (complex II) and succinate-cytochrome $c$ reductase (integrated complex II and III) were assayed as described previously (Degli Esposti \& Lenaz, 1982; Estornell et al. 1992; Lenaz et al. $1995 \mathrm{~b})$ after $10 \mathrm{~min}$ preincubation with succinate (1.25 mM) to activate complex II. Ubiquinol-cytochrome $c$ reductase (complex III) and cytochrome $c$ oxidase (complex IV) activities were measured as have been previously reported (Degli Esposti \& Lenaz, 1982). Additionally, complex I and II activities were indirectly estimated from the application of the pool equation of Kröger and Kligenberg, which has been found to be a more reliable estimation of their activity than the direct specific measurement (Lenaz et al. 1995a; Genova et al. 1995; Estornell et al. 1997).

\section{Respiratory enzyme and cytochrome contents in mitochondria}

The cytochrome content was measured in mitochondrial samples diluted at $2-3 \mathrm{mg} / \mathrm{ml}$ in potassium phosphate buffer $(50 \mathrm{mM}$; pH 7.4), EDTA (1 mM), deoxycholate (1\%), according to Vanneste (1966) and Nicholls (1976). NADH-ferricyanide reductase activity was used to estimate the content of active complex I in mitochondria (Estornell et al. 1997). The amount of complex III was obtained by dividing cytochrome $b$ content by two and that of complex IV from the cytochrome $a a_{3}$ content (Genova et al. 1995).

\section{Ultrastuctural studies}

Rat tissues were processed for electron microscopy as described by Sancho-Tello et al. (1987). The sample fragments were immediately prefixed in ice cold glutaraldehyde $(1.5 \%)$ and formaldehyde $(1 \%)$ in cacodylate buffer $(0.1 \mathrm{M}$; $\mathrm{pH} 7.4)$ for $15 \mathrm{~min}$, cut into smaller fragments (about $1 \mathrm{~mm}^{3}$ ) and fixed for $2 \mathrm{~h}$. After washing in cacodylate buffer $(0.1 \mathrm{M} ; \mathrm{pH} 7.4)$, samples were sequentially postfixed in ice-cold solution of $\mathrm{OsO}_{4}(1 \%)$ and potassium ferricyanide $(0.8 \%)$ for $1 \mathrm{~h}$, in tannic acid $(1$ $\%$ ) for $1 \mathrm{~min}$ and in uranyl acetate (5\%) for $45 \mathrm{~min}$. Fixed samples were dehydrated in graded concentrations of ethanol and embedded in Epon. Ultrathin sections were obtained with an LKB ultramicrotome (Leica, Barcelona, Spain) and examined under a Zeiss EM 10-C electron microscope (Zeiss-Germany, Ober Kochen, Germany).

\section{Statistics}

Values in the tables are presented as means and standard errors for the number of animals indicated. For Table 4 significance of differences with respect to the control group was evaluated by the unpaired $t$ test and accepted when $P<0.05$. For Tables $1-3$, a one-way ANOVA was performed. The null hypothesis was accepted for all values of those sets in which $F$ was non-significant at the level of $P<0.05$ and the sets of data in which $F$ was significant were examined by the Dunnett's multiple comparison test at $P<0.05$. The $\mathrm{CV}$ for any of the different analytical procedures employed was not higher than $5 \%$. The CV for each measurement within any experimental group ranged from 4 to $50 \%$, with the exceptions of serum retinol in vitamin A-deficient group and NADH oxidase in recovered group, which were $63 \%$ and $55 \%$, respectively.

\section{Results \\ Retinoid and tocopherol concentrations in plasma and tissues}

The concentration of retinol in serum from control, vitamin A-deficient and vitamin A-recovered rats is shown in Table 1. Rats fed on vitamin A-free diet from their birth (through the dam's milk) to $50 \mathrm{~d}$ old (vitamin A-deficient rats) showed less than $20 \%$ of control serum vitamin A concentration. When vitamin A-deficient rats were refed a control diet over $15 \mathrm{~d}$ (recovered rats) retinol values were similar to that found in the control group. Retinyl palmitate, other retinyl esters and carotenoids were not detected in serum. In contrast, retinyl palmitate was the main retinoid in liver and heart with no detectable retinol or carotenoid. The absence of carotenoids was in agreement with the composition of the diet as it does not contain any. The average amount of retinyl palmitate per gram of rat tissue determined in control liver and heart was $165 \mu \mathrm{g} / \mathrm{g}$ and $24 \mu \mathrm{g} / \mathrm{g}$ respectively. In parallel with plasma retinol, these values decreased significantly to about $5 \%$ and $30 \%$ of 
Table 1. Serum retinol and coenzyme $Q$ content in liver and heart from control, vitamin A-deficient and vitamin A-recovered rats $\dagger$ (Data are means and standard errors from eight control rats, eight vitamin A-deficient rats and five recovered rats)

\begin{tabular}{|c|c|c|c|c|c|c|}
\hline & \multicolumn{2}{|c|}{ Control } & \multicolumn{2}{|c|}{ Vitamin A-deficient } & \multicolumn{2}{|c|}{ Vitamin A-recovered } \\
\hline & Mean & SE & Mean & SE & Mean & SE \\
\hline $\begin{array}{l}\text { Serum retinol }(\mu \mathrm{M}) \\
\text { Coenzyme } Q \text { in liver }(\mathrm{nmol} / \mathrm{mg})\end{array}$ & 1.47 & 0.10 & $0.27^{*}$ & 0.06 & 1.37 & 0.05 \\
\hline Whole tissue & 0.51 & 0.07 & $0.83^{*}$ & 0.04 & 0.66 & 0.09 \\
\hline Mitochondria & $2 \cdot 4$ & $0 \cdot 2$ & $4 \cdot 6^{\star}$ & 0.4 & 3.0 & 0.4 \\
\hline Coenzyme $Q$ in heart $(\mathrm{nmol} / \mathrm{mg})$ & & & & & & \\
\hline Whole tissue & 0.50 & 0.09 & 0.54 & 0.07 & 0.47 & 0.10 \\
\hline Mitochondria & $3 \cdot 7$ & 0.3 & $5 \cdot 0^{*}$ & 0.4 & 3.8 & 0.4 \\
\hline
\end{tabular}

* Significant differences compared with controls $P<0.05$.

$\dagger$ See the text for details of the assay.

control values in vitamin A-deficient liver and heart. Control diet refed animals recovered even higher tissue retinyl palmitate levels than the control group, especially in liver (data not shown).

Plasma tocopherol levels did not change between the experimental groups as deeply as retinoids did. Although moderate variations in the amount of plasma and tissue $\alpha$ tocopherol between groups were observed, the differences did not appear significant under our sample number and assay conditions (data not shown).

\section{Coenzyme $Q$ content in liver and heart}

The amount of CoQ in whole liver was $60 \%$ higher in vitamin A-deficient rats than in control rats. CoQ content increased twofold in liver mitochondria of vitamin Adeficient rats when compared with control rats. Vitamin A deficiency also induced an increase on the CoQ concentration in heart mitochondria; however, values in whole tissue were similar to those found in control rats as previously described (Estornell et al. 1997). The amount of CoQ was restored to control levels when deficient rats were refed a control diet (Table 1).

Respiratory chain activities in liver and heart mitochondria

Tables 2 and 3 show the individual and integrated respiratory activities in liver and heart mitochondria, respectively. It is important to note that the direct assays of complex I and complex II with externally added ubiquinone analogues often underevaluate their activities. In our study, both specific activities showed non-significant differences between control and vitamin A-deficient rats (results not shown). Instead, possible changes are better assessed by the indirect calculation based on the CoQ behaviour as an homogeneous pool suggested previously (Lenaz et al. 1995a; Genova et al. 1995). Therefore, we have given in the tables those values that take the advantage of a more reliable estimation of the activity of these respiratory complexes in both liver (Lenaz et al. 1995a) and heart mitochondria (Estornell et al. 1997).

As shown in Table 2 the integrity of the respiratory chain was maintained in the liver of vitamin A-deficient rats with non-significant changes in the enzymatic activities measured. Complex I and NADH oxidase (integrated aerobic oxidation) activities were increased in liver mitochondria from recovered rats. Data from heart mitochondria (Table 3) showed that complex I and complex II were decreased in vitamin A-deficient rats when compared with control rats. Other respiratory chain activities were not changed. The recovered group showed normal respiratory activities.

\section{Respiratory enzyme and cytochrome c contents in liver and heart mitochondria}

In order to better define the alterations in the respiratory

Table 2. Specific respiratory chain activities in liver mitochondria from control, vitamin A-deficient and recovered rats $†$ (Data are means and standard errors of triplicate determinations from eight control rats, eight vitamin A-deficient rats and five recovered rats)

\begin{tabular}{|c|c|c|c|c|c|c|}
\hline \multirow[b]{3}{*}{ Activity } & \multicolumn{6}{|c|}{ Specific activity (nmol/min/mg) } \\
\hline & \multicolumn{2}{|c|}{ Control } & \multicolumn{2}{|c|}{ Deficient } & \multicolumn{2}{|c|}{ Recovered } \\
\hline & Mean & $\overline{\mathrm{SE}}$ & Mean & $\overline{\mathrm{SE}}$ & Mean & SE \\
\hline NADH oxidase & 44 & 1 & 43 & 2 & $56^{\star}$ & 3 \\
\hline $\mathrm{NADH}-\mathrm{CoQ}$ reductase & 70 & 1 & 62 & 5 & $97^{\star}$ & 7 \\
\hline Succinate-CoQ reductaseł & 74 & 8 & 75 & 4 & 67 & 12 \\
\hline Succinate-cytochrome $c$ reductase & 90 & 10 & 95 & 9 & 82 & 9 \\
\hline Ubiquinol-cytochrome $c$ reductase & 240 & 19 & 245 & 27 & 246 & 28 \\
\hline Cytochrome $c$ oxidase & 352 & 40 & 350 & 53 & 379 & 51 \\
\hline
\end{tabular}

* Significant differences compared with controls $P<0.05$

$\dagger$ See the text for details of the assay of each activity.

$\ddagger$ Activities calculated from the CoQ pool equation (Lenaz et al. 1995a). 
Table 3. Specific respiratory chain activities in heart mitochondria from control, vitamin A-deficient and recovered rats†

(Data are means and standard errors of triplicate determinations from eight control rats, eight vitamin A-deficient rats and five recovered rats)

\begin{tabular}{|c|c|c|c|c|c|c|}
\hline \multirow[b]{3}{*}{ Activity } & \multicolumn{6}{|c|}{ Specific activity (nmol/min/mg) } \\
\hline & \multicolumn{2}{|c|}{ Control } & \multicolumn{2}{|c|}{ Deficient } & \multicolumn{2}{|c|}{ Recovered } \\
\hline & Mean & SE & Mean & SE & Mean & SE \\
\hline NADH oxidase & 153 & 23 & 149 & 26 & 145 & 36 \\
\hline $\mathrm{NADH}-\mathrm{CoQ}$ reductase & 349 & 30 & $230^{*}$ & 33 & 271 & 33 \\
\hline Succinate-CoQ reductase $\neq$ & 187 & 10 & $121^{*}$ & 6 & 157 & 13 \\
\hline Succinate-cytochrome $c$ reductase & 222 & 16 & 208 & 24 & 209 & 8 \\
\hline Ubiquinol-cytochrome $c$ reductase & 618 & 37 & 756 & 60 & 652 & 25 \\
\hline Cytochrome $c$ oxidase & 739 & 74 & 757 & 96 & 756 & 132 \\
\hline
\end{tabular}

* Significant differences compared with controls $P<0.05$.

$\dagger$ See the text for details of the assay of each activity.

$\ddagger$ Activities calculated from the CoQ pool equation (Lenaz et al. 1995a).

chain promoted by the vitamin A deficiency, the respiratory-enzyme content and the amount of cytochrome $c$ were evaluated in liver and heart mitochondria (Table 4). Vitamin A-deficient rats preserved these respiratory components in liver mitochondria, although the content of complex I showed a tendency to descend. However, complex I and cytochrome $c$ contents were decreased in heart mitochondria from vitamin A-deficient rats when compared with control rats. This decrease in the level of cytochrome $c$ could be a limiting factor for the overall rate of respiration by slowing the electron-transfer rate between complex III and IV. The complex I deficiency found in heart mitochondria was well correlated with the diminished activity of complex I shown in this tissue. Complex III and IV did not change in vitamin A-deficient rats when compared to control rats.

\section{Ultrastructural studies}

Some structures similar to the multivesicular lysosomes described by Leo et al. (1983) were found in the vitamindeficient liver by electron microscopy. However, no appreciable difference was observed between control and

Table 4. Complex III, complex IV and cytochrome $c$ contents (pmol/mg) in liver and heart mitochondria from control and vitamin A-deficient rats

(Data are means and standard errors of duplicate determinations from eight control rats and eight vitamin A-deficient rats)

\begin{tabular}{lccccc}
\hline & \multicolumn{2}{c}{ Control } & & \multicolumn{2}{c}{ Deficient } \\
\cline { 2 - 3 } \cline { 5 - 6 } & Mean & SE & & Mean & SE \\
\hline Liver & $8 \cdot 4$ & $1 \cdot 0$ & & $6 \cdot 7$ & 1.0 \\
Complex I & 78 & 9 & & 77 & 10 \\
Complex III & 110 & 7 & & 119 & 16 \\
Complex IV & 108 & 10 & & 97 & 13 \\
Cytochrome $c$ & 14.8 & $1 \cdot 0$ & & $11 \cdot 3^{*}$ & 0.9 \\
Heart & 204 & 27 & & 211 & 31 \\
Complex I & 483 & 46 & & 512 & 54 \\
Complex III & 248 & 24 & $176^{*}$ & 13 \\
Complex IV & & & & \\
Cytochrome $c$ & & & & & \\
\hline
\end{tabular}

* Significant differences compared with controls $P<0.05$ vitamin A-deficient rats in the ultrastructural morphology of liver and heart mitochondria. Therefore, it appeared that the changes induced by our period of vitamin A deficiency in the respiratory chain function and composition were not reflected yet in the structure of the mitochondria. This could partially justify the reversibility of the alterations detected in the respiratory chain.

\section{Discussion}

Among the different properties of vitamin $\mathrm{A}$, the antioxidant role of some of their compounds, known for decades, has been reinvestigated in recent years in chemical as well as in biological systems. Its hydrophobic chain of polyene units can stabilize peroxyl radicals, neutralise thiyl radicals and quench singlet oxygen. In the lipophilic compartment of membranes and lipoproteins where lipid soluble vitamins are localised, retinol can act as an effective peroxyl radical scavenger. In fact, it has been shown in model phosphatidylcholine liposomes that the relative antioxidant efficiency of retinol is similar to that of tocopherol, whose antioxidant activity is well documented (Packer \& Landvik, 1990; Burton, 1994; Traber \& Packer, 1995), when radicals are generated inside a lipophylic environment. Possibly the shorter polyene chain of retinol supplies it with higher mobility to interact with radicals (Tesoriere et al. 1993; Palace et al. 1999).

In a previous work we reported that the deficiency of vitamin A induced a decrease in both the activity and the amount of the respiratory complex I (NADH: CoQ reductase) (Estornell et al. 1997) in heart mitochondria. Complex I is the largest proton-translocating enzyme of mitochondria and it contains the highest number of mitochondrial-encoded subunits. Mitochondria have been recognised as a major physiological source and target for reactive oxygen species, which arise as a consequence of oxygen reduction. The presence of several electron carriers and polyunsaturated fatty acid rich membranes makes this organelle highly susceptible to changes in the levels of physiological antioxidants (i.e. retinol, tocopherol, etc.) and to free-radical attack leading to alterations of its structural integrity and function. 
Our study shows that the respiratory-chain integrity in heart mitochondria is highly dependent on vitamin A. The vitamin A deficiency promoted a damage in the respiratory chain at different levels, being its flavin-dehydrogenase complexes the most affected. The defect in complex II shown here and also the reduced cytochrome $c$ content, which acts as mobile electron carrier between complex III and IV, must be added to the defective complex I previously described (Estornell et al. 1997). The damage induced by vitamin A deficiency in heart mitochondria, however, was not seen in liver mitochondria which preserved the integrity of its electron transfer components in the absence of vitamin A. This is in concordance with the fact that heart is a more aerobic tissue than the liver and thus, it is more exposed to oxidative injuries. Other authors have also reported tissue-different responses to damage induced by vitamin A deficiency, the liver being less affected than proliferating epithelial tissues (Evarts et al. 1995). Moreover, several oxidative treatments induced lipid membrane peroxidation in vivo in different tissues (i.e. brain, heart, etc.) but not in liver, suggesting that it has more effective antioxidant systems than the other tissues (Ciaccio et al. 1993). However, the lack of changes in respiratory chain in liver after vitamin A depletion does not imply that other manifestations related to oxidative stress could not be found in this tissue. In fact, we have found that free-radical mechanisms appear to be implicated in the mtDNA damage, mitochondrial glutathione depletion and the fall in mitochondrial membrane potential induced by vitamin A deficiency in rat liver (Barber $\mathrm{T}$ et al., Free Radical Biology and Medicine, in press).

Interestingly, the increase in the CoQ content that we have found in the heart mitochondria of vitamin A-deficient rats could maintain their overall rate of aerobic $\mathrm{NADH}$ oxidation and counteract the defects seen in the different components of their respiratory chain. This is in accordance with our previous data and with the kinetic behaviour of the CoQ pool. In kinetic terms, it is possible to increase respiratory-chain activity from NADH oxidation by increasing CoQ content over the normal level due to its lack of saturation for enzyme activities (Estornell et al. 1992). Thus, partial enzyme deficiencies can be counteracted by higher CoQ levels (Lenaz et al. 1995b; Estornell et al. 1997). An increase in mitochondrial CoQ concentration has also been described in other situations with reduced mitochondrial activity such as liver-induced cirrhosis in human and rats as a compensatory response of mitochondrial respiratory assembly for maintaining liver function (Morimoto et al. 1985; Krahenbuhl \& Reichen, 1992).

The changes in the respiratory function of mitochondria induced by vitamin A deficiency were not manifested in alterations of their structure as observed at the electron microscope. One reason for that could be that the deficiency period was not long enough for the functional deficiencies being translated in derrangements of mitochondrial morphology. Alternatively, the impairment of the function could not be severe enough. In this sense, no morphological changes were observed at light microscope level despite a $60 \%$ inhibition of complex I activity in an animal model with induced mitochondrial myopathy
(Cooper et al. 1988). However, Thomas et al. (1993) in a neuromuscular myopathy induced by feeding rats on a vitamin E-deficient diet (vitamin $\mathrm{E}$ is a known membrane antioxidant) for 1 year, found decreases of 60 and $50 \%$ in complex I and IV activities respectively, together with nonspecific degenerative changes of muscle mitochondria and disorganisation of their cristae. In agreement with our study, Leo et al. (1983) did not find any change in the structure of liver mitochondria after feeding rats on a vitamin A-deficient diet for $60 \mathrm{~d}$ and Morre et al. (1981) described modifications of the Golgi apparatus as the only consistent ultrastructural response in liver to changes in vitamin A status. The lack of structural degeneration in our condition of vitamin A deficiency probably facilitates the complete restoration of the functional changes after refeeding on the control diet.

It is known that a variety of key events in apoptosis focus on mitochondria and include changes in electron transport activity, decreased cytochrome $c$ content inside mitochondria, increased release of cytochrome $c$ to the cytosol and altered cellular oxidation-reduction status (Liu et al. 1996; Green \& Reed, 1998). It is also known that vitamin A and its metabolites are inhibitors of apoptosis (Semba, 1994; Maden et al. 1998). In our work, vitamin A deficiency impaired the integrity of heart mitochondrial function in association with a decreased cytochrome $c$ content and changes in the levels of CoQ, a physiological antioxidant in mitochondrial membrane. Moreover, in a recent work (Barber T et al., Free Radical Biology and Medicine, in press) we have shown that vitamin A deficiency induced oxidative stress, accompanied with an increase in malondialdehyde and 8-oxo-dihydro- $2^{\prime}$-deoxyguanosine (which indicate damage to mtDNA) and a drop in the mitochondrial membrane potential. All of these parameters are different signals described on the pathway in physiological cell death (Green \& Reed, 1998) and agree with data reported previously by others on vitamin A deficiency. In testis from vitamin A-deficient rats most of the germ cells from seminiferous tubules degenerated by apoptosis (Akmal et al. 1998) and, in a rat model, maternal vitamin A deficiency was correlated with placental apoptosis and perturbed fetal development (Lea et al. 1999). Moreover, in regenerating rat liver vitamin A deficiency leads to a widespread activation of nuclease activity and the administration of vitamin A to these animals enhanced the capacity of the liver to regenerate by preventing apoptosis and necrosis cell death (Evarts et al. 1995). The functional alterations found in our study may represent a pre-apoptotic stage before morphological changes become manifest. However, the mechanistic aspects of mitochondrial involvement in cell death are extremely complex and require understanding the integration of numerous mitochondrial responses that cannot be dissected easily and escapes the aim of our work.

The functional and compositional differences between the three experimental groups found in our work seem to result from the changes in retinoids rather than from those in $\alpha$-tocopherol. This suggestion is based on quantitative variations in retinoids being much more important than those in $\alpha$-tocopherol. In fact, the levels of retinyl palmitate diminished around 95 and $70 \%$ in the liver and heart of 
vitamin A-deficient animals whereas the changes in $\alpha$ tocopherol were not significant.

The present study has raised some interesting questions concerning the therapeutic use of retinol because of the high dependence for the heart mitochondrial integrity. Our results are in accordance with many experimental and epidemiological studies that are supportive of the beneficial effects of retinol as an important antioxidant for heart integrity, which is highly susceptible for free-radical attack. In fact, numerous reports have provided the basic structural and metabolic characteristics of vitamin $\mathrm{A}$ as well as information about its potential as physiological antioxidant for mitigating and defending against heart injury (for review see Palace et al. 1999). Another question is the beneficial effect of the CoQ to treat respiratory-chain diseases and also to preserve the mitochondrial integrity in ageing (Schapira, 1998; Wallace, 1999). Assuming that it is possible to incorporate CoQ into the mitochondrial membranes by external administration to patients, it could contribute to preserve the bioenergetic function in damaged mitochondria, at least if the respiratory chain complex deficiencies are not highly severe. This short-term effect of CoQ could be important in addition to the long-term effects due to its well-known antioxidant role (Shi et al. 1999). This idea opens the possibility of a combined therapeutic use of both CoQ and retinol to improve some of the heart mitochondrial diseases.

\section{Acknowledgements}

This work was supported by grants from the Fondo de Investigación Sanitaria del Ministerio de Sanidad (99/1157) and the Generalitat Valenciana (GV-D-VS-20-152-96 and GV-D-VS-20-164-96), Spain.

\section{References}

Akmal KM, Dufour JM, Vo M, Higginson S \& Kim KH (1998) Ligand dependent regulation of retinoic acid receptor alpha in rat testis: in vivo response to depletion and repletion of vitamin A. Endocrinology 139, 1239-1248.

Barber T, Triguero A, Martínez-López I, Torres L, García C, Miralles VJ \& Viña JR (1999) Elevated expression of liver gamma-cystathionase is required for the maintenance of lactation in rats. Journal of Nutrition 129, 928-933.

Barua AB \& Olson JA (1998) Reversed-phase gradient highperformance liquid chromatographic procedure for simultaneous analysis of very polar to nonpolar retinoids, carotenoids and tocopherols in animals and plant samples. Journal of Chromatography B 707, 69-79.

Burton (1994) Vitamin E: molecular and biological function. Proceedings of the Nutrition Society 53, 251-262.

Ciaccio M, Valenza M, Tesoriere L, Bongiorno A, Albiero R \& Livrea L (1993) Vitamin A inhibits doxorubicin-induced membrane lipid peroxidation in rat tissues in vivo. Archives of Biochemistry and Biophysics 302, 103-108.

Cooper JM, Petty RKH, Hayes DJ, Morgan-Hughes JA \& Clark JB (1988) Chronic administration of the oral hypoglycaemic agent diphenyleneiodonium to rats. An animal model of impaired oxidative phosphorylation (mitochondrial myopathy). Biochemical Pharmacology 37, 687-694.

Degli Esposti M \& Lenaz G (1982) Kinetics of ubiquinol-1cytochrome $\mathrm{c}$ reductase in bovine heart mitochondria and submitochondrial particles. Biochimica et Biophysica Acta 682 , 189-200.

Estornell E, Fato R, Castelluccio C, Cavazzoni M, Parenti Castelli G \& Lenaz G (1992) Saturation kinetics of coenzyme $\mathrm{Q}$ in NADH and succinate oxidation in beef heart mitochondria. FEBS Letters 311, 107-109.

Estornell E, Fato R, Pallotti F \& Lenaz G (1993) Assay conditions for the mitochondrial NADH:coenzyme Q oxidoreductase. FEBS Letters 332, 127-131.

Estornell E, Barber T \& Cabo J (1994) Improved nitrogen metabolism in rats fed on lipid-rich liquid diets. British Journal of Nutrition 71, 361-373.

Estornell E, Tormo JR \& Barber T (1997) A deficiency in respiratory complex $\mathrm{I}$ in heart mitochondria from vitamin Adeficient rats is counteracted by an increase in coenzyme Q. Biochemical and Biophysical Research Communications 233, 451-454.

Evarts RP, Hu Z, Omori N, Omori M, Marsden ER \& Thogeirsson SS (1995) Effect of vitamin A deficiency on the integrity of hepatocytes after partial hepatectomy. American Journal of Pathology 147, 699-706.

Genova ML, Castelluccio C, Fato R, Parenti Castelli G, Merlo Pich M, Formiggini G, Bovina C, Marchetti M \& Lenaz G (1995) Major changes in complex I activity in mitochondria from aged rats may not be detected by direct assay of NADH:coenzyme Q reductase. Biochemical Journal 311, $105-109$

Green DR \& Reed JC (1998) Mitochondria and apoptosis. Science 281, 1309-1312.

Johnson D \& Lardy H (1967) Isolation of liver or kidney mitochondria. Methods in Enzymology 10, 94-96.

Krahenbuhl S \& Reichen J (1992) Adaptation of mitochondrial metabolism in liver cirrhosis. Different strategies to maintain a vital function. Scandinavian Journal of Gastroenterology 193, 90-96.

Kröger A (1978) Determination of contents and redox states of ubiquinone and menaquinone. Methods in Enzymology 53, 579-591.

Lea RG, Riley SC, Antipatis C, Hannah L, Ashworth CJ, Clark DA \& Critchley HO (1999) Cytokines and the regulation of apoptosis in reproductive tissues: a review. American Journal of Reproductive Immunology 42, 100-109.

Lenaz G, Fato R, Genova ML, Formiggini G, Parenti Castelli G \& Bovina C (1995a) Underevaluation of complex I activity by the direct assay of $\mathrm{NADH}$-coenzyme $\mathrm{Q}$ reductase in rat liver mitochondria. FEBS Letters 366, 119-121.

Lenaz G, Bovina C, Castelluccio C, Cavazzoni M, Estornell E, Fato R, Huertas JR, Merlo-Pich M, Pallotti F, Parenti Castelli G \& Rauchova H (1995b) Modes of coenzyme Q function in electron transfer. Protoplasma 184, 50-62.

Leo MA, Sato M \& Lieber CS (1983) Effect of hepatic vitamin A depletion on the liver in humans and rats. Gastroenterology $\mathbf{8 4}$, 562-572.

Liu X, Kim CN, Yang J, Jemmerson R \& Wang X (1996) Induction of apoptotic program in cell-free extracts: requirement for dATP and cytochrome c. Cell 86, 147-157.

Livrea MA \& Tesoriere L (1998) Antioxidant activity of vitamin A within lipid environments. In Subcellular Biochemistry, pp. 113-143 [PJ Quinn and VE Kagan, editors]. New York: Plenum Press.

Maden M, Gale E \& Zile M (1998) The role of vitamin A in the development of the central nervous system. Journal of Nutrition 128, 471S-475S.

Morimoto T, Jikkoh A, Yokoo N, Taki Y, Tanaka J, Kamiyama Y, Ozawa K \& Tobe T (1985) Changes in coenzyme Q level in mitochondria of cirrhotic rat liver. Life Sciences 36, 15771580 . 
Morre DM, Morre DJ \& Walter M (1981) Vitamin A effects on hepatic Golgi apparatus architecture. European Journal of Cellular Biology 25, 28-35.

Nicholls P (1976) Catalytic activity of cytochromes $c$ and $c_{1}$ in mitochondria and submitochondrial particles. Biochimica et Biophysica Acta 430, 30-45.

Packer L \& Landvik S (1990) Vitamin E in biological systems. Advances in Experimental Medicine and Biology 264, 93-103.

Palace VP, Khaper N, Qin Q \& Singal PK (1999) Antioxidant potentials of vitamin A and carotenoids and their relevance to heart disease. Free Radical Biology and Medicine 26, 746-761.

Pasatiempo AM, Taylor CE \& Ross AC (1991) Vitamin A status and the immune response to pneumococcal polysaccharide: effects of age and early stages of retinol deficiency in rats. Journal of Nutrition 121, 556-562.

Reeves PG, Nielsen FH \& Fahey GC (1993) AIN-93 purified diets for laboratory rodents: final report of the American Institute of Nutrition ad hoc writing Committee on the reformulation of the AIN-76 rodent diet. Journal of Nutrition 123, 1939-1953.

Ross AC (1992) Vitamin A status: relationship to immunity and the antibody response. Proceedings of the Society for Experimental Biology and Medicine 200, 303-320.

Sancho-Tello M, Renau-Piqueras J, Báguena-Cervellera R \& Guerri C (1987) A biochemical and stereological study of neonatal rat hepatocyte subpopulations. Effect of pre- and postnatal exposure to ethanol. Virchows Archives of Biology and Cellular Pathology 54, 170-181.

Schapira AHV (1998) Human complex I defects in neurodegenerative diseases. Biochimica et Biophysica Acta 1364, 261-270.

Selvaraj RJ \& Susheela TP (1970) Estimation of serum vitamin A by a microfluorometric procedure. Clinica Chimica Acta 27, $165-170$.
Semba RD (1994) Vitamin A, immunity, and infection. Clinical Infectious Diseases 19, 489-499.

Semba RD (1999) Vitamin A and immunity to viral, bacterial and protozoan infections. Proceedings of the Nutrition Society $\mathbf{5 8}$, 719-727.

Shi H, Noguchi N \& Niki E (1999) Comparative study on dynamics of antioxidative action of alpha-tocopherol hydroquinone, ubiquinol and alpha-tocopherol against lipid peroxidation. Free Radical Biology and Medicine 27, 334-346.

Smith AL (1967) Preparation, properties and conditions for assay of mitochondria: slaughterhouse material, small scale. Methods in Enzymology 10, 81-86.

Tesoriere L, Ciaccio M, Bongiorno A, Riccio A, Pintaudi AM \& Livrea MA (1993) Antioxidant activity of all-trans-retinol in homogeneous solution and in phosphatidylcholine liposomes. Archives of Biochemistry and Biophysics 307, 217-223.

Thomas PK, Cooper JM, King RH, Workman JM, Schapira AHV, Goss-Sampson MA \& Muller DPR (1993) Myopathy in vitamin E deficient rats: muscle fibre necrosis associated with disturbances of mitochondrial function. Journal of Anatomy 183, 451-461.

Traber MG \& Packer L (1995) Vitamin E: beyond antioxidant function. American Journal of Clinical Nutrition 62, 1501S1509S.

Vanneste VH (1966) Molecular proportion of the fixed cytochrome components of the respiratory chain of Keilin-Hartree particles and beef heart mitochondria. Biochimica et Biophysica Acta 113, 175-178.

Wallace DC (1999) Mitochondrial diseases in man and mouse. Science 283, 1482-1488.

Zile M (1998) Vitamin A and embryonic development: an overview. Journal of Nutrition 128, 455S-458S. 